\title{
Control of Prosodic Focus in Corpus-based Generation of Fundamental Frequency based on the Generation Process Model
}

\author{
Keiko Ochi ${ }^{1}$, Keikichi Hirose ${ }^{1}$, and Nobuaki Minematsu ${ }^{2}$ \\ ${ }^{1}$ Department of Information and Communication Engineering, the University of Tokyo, Tokyo \\ ${ }^{2}$ Department of Electrical Engineering and Information Systems, the University of Tokyo, Tokyo \\ \{Ochi, hirose, minematsu\}@gavo.t.u-tokyo.ac.jp
}

\begin{abstract}
A method was developed for generating sentence $F_{0}$ contours, when a focus is placed in one of bunsetsu of an utterance. The method is to predict differences in $F_{0}$ model commands between with and without focus utterances, and applies them to the F0 model commands predicted beforehand by the baseline method. The validity of the method was proved by the experiment on $F_{0}$ contour generation and speech synthesis.

Index Terms: speech synthesis, $F_{0}$ contour, Focus
\end{abstract}

\section{Introduction}

In spontaneous speech, speakers may frequently place focuses on selected word(s) in their utterances. Therefore, focus control is becoming one of important issues in spontaneous speech synthesis. However, given a speech synthesis system without specific focus control, it is not efficient to prepare a large speech corpus with focus control and train the speech synthesis system from the beginning. While we have developed a corpus-based method of synthesizing $F_{0}$ contours in the framework of the generation process model ( $F_{0}$ model) [1] and realized speech synthesis in reading and dialogue styles with various emotions [2, 3]. It is rather easy to analyze the prosodic controls obtained by statistical methods and to modify generated $F_{0}$ contours in another corpus-based way, which is trained using a small speech corpus.

In this paper, we propose a method of realizing prosodic focus as a supplemental process to our corpus-based method of $F_{0}$ contour generation; train binary decision trees for differences in phrase command magnitudes and accent command amplitudes between utterances with and without focuses. The command values predicted by our baseline method (for utterances without specific focuses) are modified using the differences. By concentrating to the differences, a better training for $F_{0}$ change due to focal position comes possible only with a limited speech corpus. Moreover, speakers for the training need not be the same for those of the baseline.

\section{Method and Result}

Observations of $F_{0}$ contours imply that we can represent $F_{0}$ change due to focal positions as changes in $F_{0}$ model command magnitudes/amplitudes. The differences in phrase command magnitudes are predicted using CART with inputs of distance from focused bunsetsu, position of current bunsetsu in phrase/sentence, mora numbers and accent types, distance in mora from the preceding phrase command, depth of syntactic boundary, and pause between preceding and current bunsetsus. The differences in accent command amplitudes are also predicted in a similar way. 172 utterances by a female speaker were used for the prediction. Figure 1 shows an example of generated $F_{0}$ contour when the predicted changes are applied to $F_{0}$ model parameters predicted by the baseline method. Result of preliminary perceptual experiment confirmed that the focus was perceived on the right place by the method.

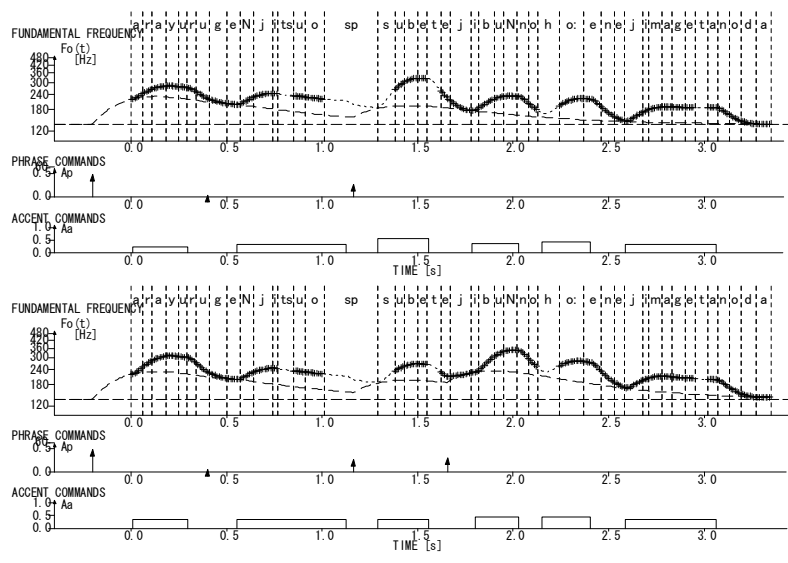

Figure 1: Generated $F_{0}$ contours and $F_{0}$ model parameters of Japanese sentence "arayuru genjitsuo subete jibunno hooe nejimagetanoda (They bended all the realities to their side)". The first and second panels show when "subete" and "jibunno" are focused, respectively.

\section{Conclusion}

A method was developed to generate $F_{0}$ contours with prosodic focuses. The method works only with an additional small speech corpus with various focal positions. Detailed results including variations of applying predicted differences to $F_{0}$ model commands will be shown in the presentation.

\section{References}

[1] Hirose, K., Sato, K., Asano, Y., and Minematsu, N., "Synthesis of $F_{0}$ contours using generation process model parameters predicted from unlabeled corpora: Application to emotional speech synthesis," Speech Communication, Vol.46, Nos.3-4, pp.385-404 (2005-7).

[2] Hirose, K., Ochi, K., and Minematsu, N., "Corpus-based generation of prosodic features from text based on generation process model," Proc. Interspeech, pp.1274-1277 (2007-8).

[3] Fujisaki, H. and Hirose, K., "Analysis of voice fundamental frequency contours for declarative sentences of Japanese," $J$. Acoust. Soc. Japan (E), Vol.5, No.4, pp.233-242 (1984-10). 